\title{
A capillary electrophoresis method for the determination of selected biogenic amines and amino acids in mammalian decomposition fluid
}

\author{
Swann, L.M., Forbes, S.L. and Lewis, S.W.* \\ ${ }^{a}$ Department of Chemistry, Curtin University of Technology, Box U1987 Perth Western \\ Australia 6845 \\ ${ }^{b}$ Faculty of Science, University of Ontario Institute of Technology, Oshawa, Ontario, \\ Canada, $\mathrm{L} 1 \mathrm{H} 7 \mathrm{~K} 4$
}

*author for correspondence, s.lewis@curtin.edu.au

\begin{abstract}
A simple capillary zone electrophoresis method for the determination of selected biogenic amines (tyramine and tryptamine) and amino acids (tryptophan, phenylalanine and tyrosine) in mammalian decomposition fluids is presented. Separations were carried out in a fused silica capillary ( $75 \mu \mathrm{m}$ i.d., total length $65 \mathrm{~cm}$, effective length $56 \mathrm{~cm}$ ) with detection by ultraviolet absorbance spectrophotometry at $200 \mathrm{~nm}$. In order to improve resolution and total analysis time, the method was subjected to optimisation utilising a chemometric approach. A screening design was carried out followed by a central composite design (CCD), using peak resolution and total analysis time as response factors. The influences of four experimental variables $(\mathrm{pH}$, background electrolyte concentration, percentage of organic modifier (methanol) and applied voltage) were investigated. Optimum separation conditions were determined to be; a background electrolyte of boric acid (70 $\mathrm{mM})$ adjusted to $\mathrm{pH} 9.5$ with $0.1 \mathrm{M}$ sodium hydroxide with $32 \%$ methanol (v/v). Applied voltage was $30 \mathrm{kV}$, with the resulting current being less than $26 \mu \mathrm{A}$. Under these conditions the analytes were separated within 12 minutes. Tryptamine, tyramine, tryptophan, tyrosine and phenylalanine were identified by migration time and spiking in porcine decomposition fluids.
\end{abstract}


Keywords: capillary electrophoresis, decomposition, biogenic amines, central composite design, forensic science

\section{Introduction}

Decomposition of mammalian soft tissue is a post-mortem process that, depending on environmental conditions and physiological factors, will proceed until complete disintegration of the tissue [1]. The major stages of decomposition involve complex reactions which result in the chemical breakdown of the body's main constituents; lipids, proteins, and carbohydrates [2]. As decomposition proceeds, these macromolecules degrade to their structural components that include amino acids, fatty acids, and glucose [3].

The decomposition process is highly variable and complex in nature, making it notoriously difficult to estimate time of death. Scientific evidence of time of death is preferable to circumstantial information or witness testimony, which are vulnerable to mistakes or deception. Scientifically measured parameters that can be used to estimate time of death may be obtained from the body and its immediate surroundings $[4,5]$.

It has been suggested that measurement of chemical parameters may also be useful in establishing post-mortem interval based upon the temporal variation of certain chemical biomarkers [3]. Previous analytical work by the authors into the chemical compounds produced during the course of the decomposition of an animal model has shown a variety of compounds to be present, including short and long chain fatty acids, as well as cyclic amides $[6,7]$. The biodegradation pathways of lipids, proteins and carbohydrates $[8,9]$ leads to the expectation that amino acids and biogenic amines would be present in decomposition fluid. 
Capillary electrophoresis (CE) has frequently been applied to the separation and detection of nitrogenous compounds in a wide variety of matrices, particularly within the forensic and pharmaceutical industry [10-12]. It offers the potential for highly efficient and rapid separations, and thus is extremely useful when analyzing complex mixtures. CE can cope with analytes that are not suitable for gas chromatography (GC) due to thermal instability, while also providing more rapid separations than are achievable with liquid chromatography (LC), whilst still maintaining high resolution [13]. In addition CE is well able to cope with 'dirty' or complex sample matrices, such as alcoholic beverages and food, particularly cheese and fish [14-17].

CE has been applied to separations of biogenic amines in environmental samples, foods and beverages, with derivatisation of the amines often being necessary to enable detection [14-18]. The use of micelles is often employed [18, 19] or complex buffer systems are needed $[16,18]$ to achieve satisfactory analyte mobility, often with extended analysis times $[14,15,20]$. Zhang et al. determined seven biogenic amines in soy sauce, fish and wine samples by micellar electrokinetic chromatography (MEKC) with laser induced fluorescence detection after derivatisation of the amines with the fluorescent label 3-(4-fluorbenzoyl)-2-quinolinecarboxaldehyde [17]. Krizek and Pelikanova separated biogenic amines as $\mathrm{N}$-substituted benzamide derivatives in fish meat by MEKC with UV-visible detection [15]. Liu and Cheng analysed lake water using MEKC with post-column chemiluminescence detection of $\mathrm{N}$-(4-aminobutyl)-N-ethylisoluminol labeled amines. Direct analysis of amines in selected food samples without derivatisation has been achieved using separation on a fluorinated ethylene-propylene co-polymer analytical capillary with conductivity detection [18].

Amines have also been separated by $\mathrm{CE}$ without derivatisation using an ammonium acetate buffer containing acetonitrile or methanol as an organic modifier at $\mathrm{pH} 7.5$. Separation was found to be best achieved with acetonitrile as the modifier, however there was no application to any real samples [21]. Cortacero-Ramirez et al. analysed bottled 
beer for 18 amines and amino acids without the need for derivatisation or micelles, however, there was not complete baseline resolution of all the compounds of interest. Separation took over 30 minutes to achieve [20].

Taking the above considerations into account, CE should be well suited to the determination of biogenic amines in the complex matrix of decomposition fluids. To the best of our knowledge, no analytical methodology using $\mathrm{CE}$ as a separation technique for the determination of the chemical species present in decomposition fluid has been reported so far.

This paper reports a rapid and simple capillary electrophoresis method for selected biogenic amines and amino acids in decomposition fluid. Due to the ethical issues involved in the use of human cadavers, pig (Sus scrofa) carcasses were used to model the human decomposition process [22]. They are considered to be an acceptable substitute due to their similarity to human torsos in weight, fat to muscle ratio, hair coverage, biochemistry and physiology $[9,23]$. The method was optimized using a screening design followed by a central composite design.

\section{Materials and Methods}

Chemicals and Reagents

Chemicals were obtained from the following suppliers: Tyramine, tryptophan and indole (Sigma-Aldrich, New South Wales, Australia), tyrosine, tryptamine and phenylalanine (Fluka, New South Wales, Australia), aniline, boric acid and sodium hydroxide (BDH, Victoria, Australia), benzylamine (ACROS, New Jersey, USA), methanol (LC Grade, 
Mallinckrodt, Missouri, USA). Standards (400 mg/L) were prepared in methanol:water (1:1). MilliQ water was prepared in-house using a Purelab water system. Peak identification was carried out by the injection of known individual standards and by spiking samples with known standards.

The background electrolyte was prepared by mixing varying concentrations of boric acid (33mM, $50 \mathrm{mM}, 75 \mathrm{mM}, 100 \mathrm{mM}, 117 \mathrm{mM})$ with $0.1 \mathrm{M}$ sodium hydroxide $(\sim 80: 20 \mathrm{v} / \mathrm{v})$; the $\mathrm{pH}$ was adjusted to the desired level with $0.1 \mathrm{M}$ sodium hydroxide. Methanol $(13.2 \%$, $20 \%, 30 \%, 40 \%, 46.8 \%)(\mathrm{v} / \mathrm{v})$ was then added to the buffer solution.

All buffer solutions and standards were degassed by filtration through a $0.45 \mu \mathrm{m}$ PTFE Gelman Acrodisc ${ }^{\circledR}$ syringe filter prior to injection and analysis.

\section{Sample collection and preparation}

The collection of decomposition fluid samples is described in a previous paper [6]. Initial studies were performed with pork rashers (belly pork, Sus scrofa) to aid in method development. Further trials were carried out with stillborn piglets and adult pig carcasses (Sus scrofa) to create a more realistic model of decomposition and to gain a more accurate understanding of the composition of decomposition fluid.

All samples were stored in the freezer prior to analysis. Samples were prepared by a 1:4 dilution in methanol, vortexed for 1 minute, followed by filtration through a $0.45 \mu \mathrm{m}$ PTFE Gelman Acrodisc $®$ syringe filter. 


\section{Instrumentation}

Experiments were performed using an Agilent 3DCE Capillary Electrophoresis system equipped with a diode array detector. An uncoated $75 \mu \mathrm{m}$ fused silica capillary of total length $65 \mathrm{~cm}$ and effective length $56 \mathrm{~cm}$ (Agilent) was used for analysis. Instrument control and data acquisition were performed using ChemStation software.

Sample introduction was achieved by pressure injection at 50 mbar for $2.5 \mathrm{secs}$. During sample analysis, a constant voltage of $30 \mathrm{kV}$ was applied, resulting in an electrophoretic current of $26 \mathrm{uA}$. The temperature around the capillary was maintained at $30^{\circ} \mathrm{C}$. For all experiments, the wavelength of detection was $200 \mathrm{~nm}$. The final optimized running buffer consisted of $70 \mathrm{mM}$ boric acid adjusted to $\mathrm{pH} 9.5$ with $0.1 \mathrm{M}$ sodium hydroxide and $32 \%$ methanol (v/v).

When a new capillary was used, it was conditioned for 30 minutes with $1.0 \mathrm{M}$ sodium hydroxide, MilliQ water and running buffer respectively. At the beginning of each day, the capillary was flushed for 5 minutes with $1.0 \mathrm{M}$ sodium hydroxide, MilliQ water and running buffer successively. For the screening design, the capillary was flushed for 10 minutes with the new running buffer (as described by conditions detailed in Table 1) between each run. Preconditioning before each run was achieved by flushing with running buffer for 3 minutes. Between runs, post-conditioning for 3 minutes with MilliQ water, 3 minutes with $0.1 \mathrm{M}$ sodium hydroxide and 3 minutes with MilliQ was performed. For the optimized system, when reproducible analysis was achieved, postconditioning was reduced to 3 minutes with MilliQ water only.

The screening design and central composite design were generated and graphed using SAS/JMP software version 7. 


\section{Results and Discussion}

The biogenic amines selected for this study are presented in Table 1. These compounds were chosen on the basis of their availability and their potential presence in decomposition fluid suggested by our previous studies [6]. On reviewing the literature $[15,17,18]$, the method of Krizek and Pelikanova [15] was selected for further study due to its relative simplicity and applicability to the analytes listed in Table 1.

\section{Table 1. Selected biogenic amines. * indicates successful separation by method outlined in this work.}

We found in a similar fashion to the original authors that the performance of the derivatisation was highly dependent on vigorous mixing of the reagent and analyte. This variability in performance ruled out this technique for application to decomposition fluids. However, while investigating the separation conditions we discovered that we could successfully separate and detect a number of the amines and amino acids (Table 1) without prior derivatisation or the presence of micelles. In comparison to the method outlined by Krizek and Pelikanova, no sample preparation was required other than a simple dilution and filtration. Samples could be prepared and ready for analysis in a matter of minutes. Initial investigation determined that two amines (tryptamine, tyramine) and three amino acids (tryptophan, phenylalanine, tyrosine) could be separated within 8 minutes using a fused silica capillary with a simple background electrolyte consisting of boric acid $(50 \mathrm{mM})$ with $25 \%$ methanol $(\mathrm{v} / \mathrm{v})$ at a $\mathrm{pH}$ of 9.4. Benzylamine and aniline co-eluted and also partially overlapped with tyramine. All of the other compounds were baseline resolved (Figure 1). Those compounds successfully separated and detected all have primary amine functionalities and a chromophoric benzene ring. A trial run with a decomposition fluid sample indicated the presence of the analytes in Table 1 except for 
benzylamine and aniline. Considering the rapid nature, lack of sample preparation and simplicity of this approach we proceeded to optimize the separation conditions in order to achieve baseline resolution.

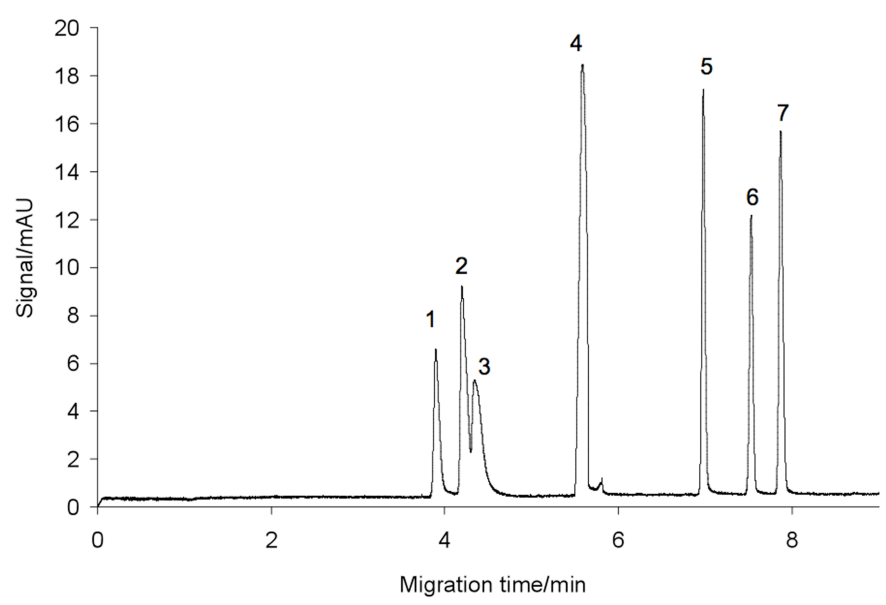

Figure 1. Unoptimised separation. Peak identification: 1. tryptamine, 2. tyramine, 3. benzylamine/aniline, 4 . neutral, 5. tryptophan, 6. tyrosine, 7. phenylalanine.

When considering optimisation of this CE method, there is the potential for interactions between factors that effect a $\mathrm{CE}$ separation. These include $\mathrm{pH}$, background electrolyte [BGE] concentration, applied voltage and percentage of organic modifier. A univariate (one-at-a-time) approach was likely to be ineffective for optimizing this separation, and as such, a multivariate chemometric approach was used. This consisted of a screening design to determine the significant individual factors and interactions, followed by a central composite design to optimize the method with respect to resolution and time.

Screening Design

To establish the appropriate experimental domain and evaluate the influence of buffer $\mathrm{pH}$, concentration of background electrolyte (boric acid), \% of organic modifier (methanol) and applied voltage, a 2-level full factorial design $\left(2^{4}\right)$ was used resulting in a 
total of 16 unique experiments. The screening design also established the significance of possible interactions between variables. All possible interactions were considered including secondary (2-way), tertiary (3-way) and quaternary (4-way) interactions.

Resolution between peaks for each electropherogram was evaluated using Equation 1:

$$
R=\frac{2\left(t_{2}-t_{1}\right)}{w_{2}+w_{1}}
$$

where $t_{2}$ and $t_{1}$ are the migration times and $w_{2}$ and $w_{1}$ are the corresponding peak widths at the bases of adjacent peak pairs [24]. For overlapping and co-eluting peaks, the resolution was estimated using data from Snyder [25] A single response objective function (Chromatographic Exponential Function; CEF, Equation 2) was used to evaluate the quality of each electropherogram with regard to resolution and time [26].

\section{Equation 2:}

$$
C E F=\left(\sum_{i=1}^{n-1}\left(1-e^{a\left(R_{o p}-R_{i}\right)}\right)^{2}+1\right)\left(1+\frac{t_{f}}{t_{\max }}\right)
$$

$R_{\text {opt }}$ and $R_{i}$ are selected optimum resolution and the resolution of the $i t h$ peak pair respectively, $t_{\max }$ and $t_{f}$ are the maximum acceptable time (15 minutes) and the elution time of the final peak respectively, $a$ is the slope adjustment factor (default $=1.5$ ) and $n$ is the number of expected peaks. The design matrix for the factors and their high $(+1)$ and low values (-1), along with the calculated response factors, are summarized in Table 2. Experiments were run in a randomized order (using random number tables generated in MS Excel) but have been shown sequentially for ease of viewing. 
Table 2: Levels of the four design variables as used in the screening design. (-1) indicates the low level, $(+1)$ indicates the high level. Table 1 also details the components of the running buffers.

The calculated CEF value was input into the screening design analysis tool using JMP version 7 software. A high CEF value indicates a poorly resolved electropherogram, therefore, $\mathrm{CEF}$ was optimized to a minimum. The evaluated data showed that $\mathrm{pH}$ ( $\mathrm{p}$ value $=0.0013$ ) was the most significant individual factor with the greatest effect on the quality of the electropherogram. The significant effect of $\mathrm{pH}$ is expected in $\mathrm{CE}$ as $\mathrm{pH}$ determines the degree of ionization for moderate and weakly basic or acidic analytes [27]. Manipulation of the buffer $\mathrm{pH}$ therefore affects the electrophoretic mobilities of the analytes in question $[13,27]$. The concentration of the background electrolyte (pvalue $=0.0106$ ) was also a major factor. Voltage and the $\%$ organic modifier had the least impact ( $\mathrm{p}$-values $=0.4382$ and 0.6378 respectively), with the only apparent effect being a decrease in the analysis time at the higher voltage, although often at the expense of resolution.

The secondary interaction between $\mathrm{pH}^{*}[\mathrm{BGE}]$ was the most significant ( $\mathrm{p}$-value=0.0119) as seen in Table 3, with $\mathrm{pH}$ having a high, positive effect at both high and low concentrations of background electrolyte. Although the percentage of organic modifier did not have a significant individual effect, its secondary interaction with $\mathrm{pH}$ ( $\mathrm{p}$ value $=0.0285$ ) is of value. The $\mathrm{pH}$ had a high effect at both low and high levels of organic modifier. For this reason, the percentage of methanol added to the buffer system cannot be excluded from further optimisation studies. All of the secondary interactions involving voltage were insignificant. Voltage had little to no effect at both high and low levels of the other three factors. Voltage was deemed to have no effect on the quality of the electropherogram and was therefore excluded from the central composite design to optimise the remaining parameters. The associated $\mathrm{p}$-values for all interactions are listed in Table 3. 
Table 3: p-values for individual factors and all interactions showing statistical significance.

As expected, none of the tertiary interactions were significant statistically (p-values $>0.05$ ). The four-way interaction between all factors was also not statistically significant in terms of effect on resolution and time.

Central composite design

A central composite design (CCD) was chosen to optimise the separation of target compounds because of its usefulness in viewing the entire response surface and how a response relates to the value of various factors [28].

A 2-level, 3-factor central composite design is detailed in Table 4. The design consists of $8\left(2^{3}\right)$ factorial points at all possible combinations of \pm 1 , with 7 star points, including a central point of the form $(0,0,0,0)$ and six points of the form $( \pm 1.68,0,0,0)$, with a recommended 5 replicates in the centre of the form $(0,0,0,0)$ giving a total of 20 experiments [28]. The replicates give an estimation of the error associated with the design. All star points are equidistant from the origin and are placed at $\pm \sqrt[4]{2^{f}}$ where $\mathrm{f}$ is the number of factors, equaling 1.68 for three factors [28]. The star points correspond to $\mathrm{pH}$ values of 8.66 and 10.34 , [BGE] values of $33 \mathrm{mM}$ and $117 \mathrm{mM}$ and organic modifier at $13.2 \%$ and $46.8 \%$, at coded positions \pm 1.68 from the centre. The purpose of the star points is to test the robustness of the system. The unique coded experiments can be seen in Table 4. Experiments were randomised (using random number tables generated in MS Excel) but have been shown sequentially for ease of viewing.

Table 4: Coded three-factor central composite design consisting of three levels, detailing star points and replication. 
Using the JMP software, a response surface was generated (Figure 2). As CEF was optimized to a minimum, the minimum point on the curve was used to detail the optimum parameters for the separation conditions.

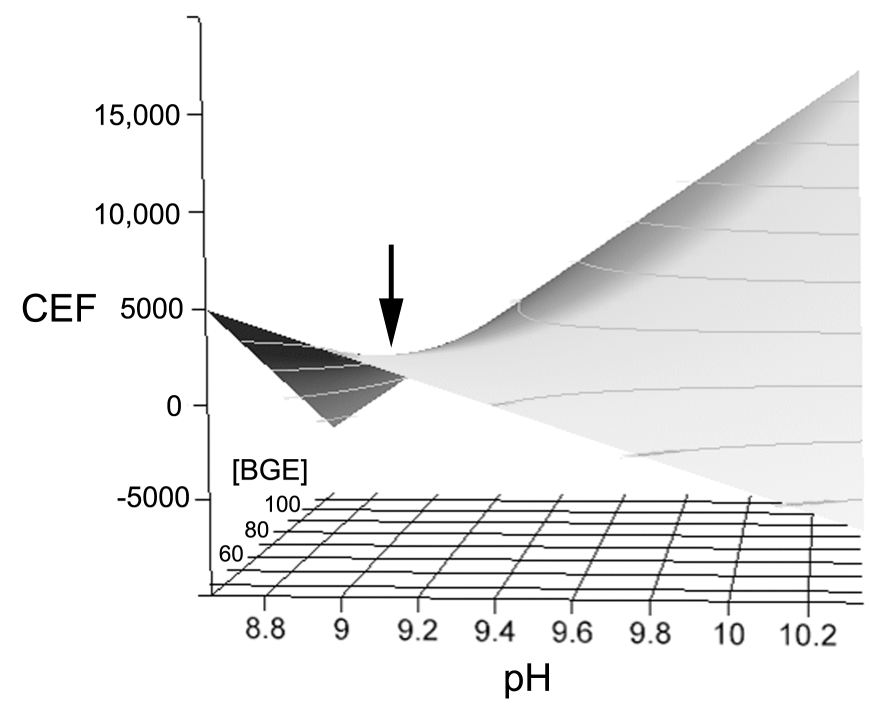

Figure 2: Response surface generated using JMP for the central composite design, optimizing CEF to a minimum. Arrow indicates optimum point on response surface

From the curve, the range of values obtained for each parameter was; $\mathrm{pH} 9.4$ - 9.6, [BGE] $65-75 \mathrm{mM}$, \% organic modifier $30-35 \%$. As the values obtained were ranges, the middle point of each was chosen as the specific optimum conditions for each factor (i.e. $\mathrm{pH}$ 9.5, [BGE] 70mM, \% MeOH 32.5\%). A comparison of a standard mixture containing eight known compounds at the pre- and post-optimum conditions is shown in Figure 3, with migration time data presented in Table 5. 


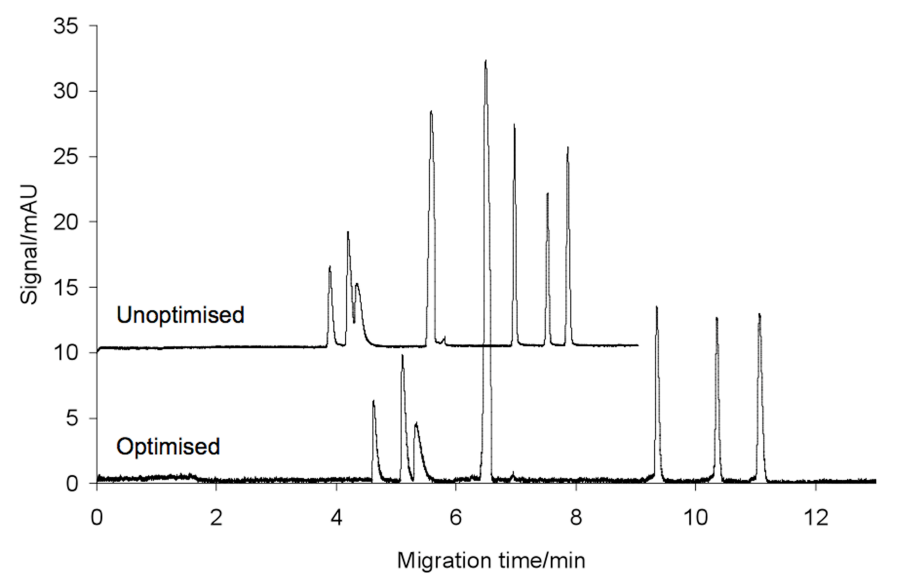

Figure 3: Comparison of the standard mixture at pre- and post-optimum conditions.

Table 5: Peak identification and migration times (mins) for standard mixture containing eight biogenic amines and amino acids at pre- and post-optimisation conditions by capillary electrophoresis.

The resolution between peaks 2 and 3 was used as the indicator for the quality of the separation. In the pre-optimised conditions, distinct separate peaks are detectable but not baseline resolved. The analysis time for this sample is less than 8 mins. In the postoptimised standard, peaks 2 and 3 are completely baseline resolved. Although the analysis time for this separation is slightly longer (12 mins), the considerable improvement in resolution is an acceptable compromise. This compares very favourably to the method of Cortacero-Ramirez et al. [20], which has an analysis time in excess of 30 minutes for similar analytes.

The optimum $\mathrm{pH}$ of the background electrolyte was established to be 9.5, and this is to be expected considering the $\mathrm{pK}_{\mathrm{a}}$ values for the successfully separated analytes (see Table 1). It would also be expected that indole would not be separated under these conditions. However, there is at this time no explanation for the co-elution of benzylamine and aniline, as the reference value for the $\mathrm{pK}_{\mathrm{a}}$ for aniline is different from the other analytes and one would not have expected any separation of this compound. 


\section{Detection Limits}

While the intention of this study is primarily qualitative, preliminary studies into whether this method could also be applied quantitatively were carried out. Mixed standards containing the selected analytes over the concentration range of $4-80 \mathrm{mg} / \mathrm{L}$ were prepared and analysed. The resulting calibration functions are presented in Table 6. Detection limits, ranged from $5.8 \mathrm{mg} / \mathrm{L}$ for tyramine to $8.6 \mathrm{mg} / \mathrm{L}$ for phenylalanine. These are comparable to those reported by Cortacero-Ramirez [20] and Lange et al. [29] who obtained detection limits in the $5.6 \mathrm{mg} / \mathrm{L}$ and $6.0 \mathrm{mg} / \mathrm{L}$ respectively using UVvisible absorbance detection. In order to apply this method quantitavely it will require the use of an internal standard. Either benzylamine or aniline would appear to be good candidates as they are absent in decomposition fluid samples, but this requires further study.

Table 6: Calibration data for selected biogenic amines

\section{Decomposition samples}

The optimized running conditions were applied to decomposition fluid samples collected as described in [6]. The complex sample matrix meant that samples were expected to have a higher signal:noise and potentially have more compounds present than in the standard mixture, thus requiring a longer analysis time. An example electropherogram produced by a decomposition fluid sample analysed using the optimised CE conditions can be seen in Figure 4. 


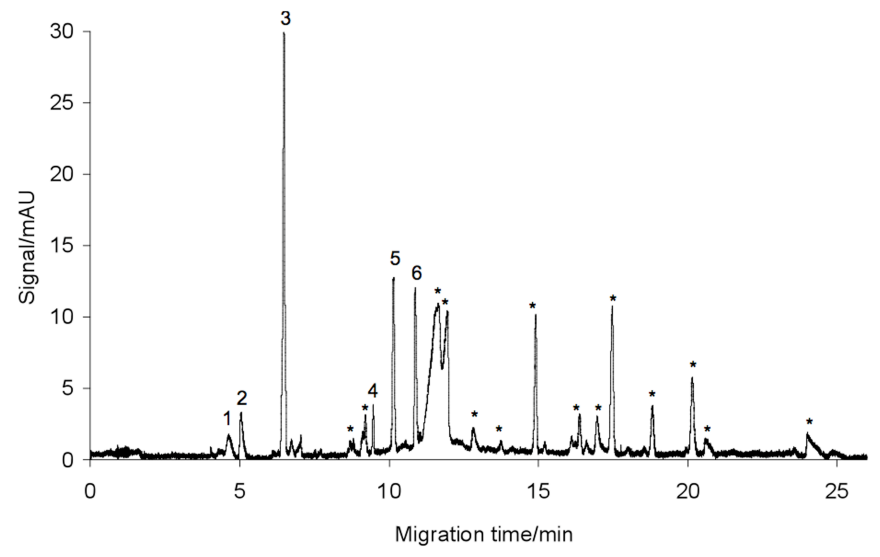

Figure 4: Electropherogram at optimized running conditions for the decomposition fluid sample. Peaks identities are: 1. tryptamine, 2. tyramine, 3. neutral, 4. tryptophan, 5. tyrosine, 6 . phenylalanine. * unidentified components.

All the compounds present in the standard mixture were detected and identified in the decomposition sample by spiking and migration time, with the exception of benzylamine/aniline. These two compounds are not expected in decomposition fluid, as there is no relevant biodegradation pathway that would suggest their presence after degradation of fats, lipids or carbohydrates $[8,9]$. A number of unidentified peaks were also observed (see Figure 4), and these will be the subjects of further study in order to investigate how they vary during the course of decomposition. Capillary electrophoresismass spectrometry and liquid chromatography-mass spectrometry will be investigated for their potential to elucidate the identity of these unknowns.

\section{Conclusions and Future Work}

A simple and rapid CE method for the determination of biogenic amines and amino acids was developed. A chemometric approach was found to be useful for rational and rapid optimization of the separation parameters. The method was successfully applied to porcine decomposition fluid samples, in which tyramine, tryptamine, phenylalanine, tryptophan and tyrosine were all identified by migration time and spiking. The method 
will be applied to the analysis of samples from a decomposition field trial to investigate the temporal variation of these compounds in mammalian decomposition fluids.

The potential also exists for application of the method to the rapid determination of tryptamine and tyramine in particular for pharmaceutical analysis and studies into food spoilage [14-16].

\section{Acknowledgments}

We gratefully acknowledge Dr. Jeff Hughes (RMIT University, Melbourne, Australia) for guidance and advice with experimental design and mathematical equations. Lisa Swann is supported by an Australian Postgraduate Award.

\section{References:}

[1] W.E.D. Evans, The Chemistry of Death, Charles C Thomas, Springfield, IL, 1963.

[2] M.A. Clark, M.B. Worrell, J.E. Pless, in: W.D. Haglund, M.H. Sorg (Eds.), Taphonomy: The postmortem fate of human remains, CRC Press, Boca Raton, 1997, p. 151-164.

[3] A.A. Vass, S.A. Barshick, G. Sega, J. Caton, J.T. Skeen, J.C. Love, J.A. Synstelien, J. Forensic Sci., 47 (2002) 542.

[4] R. Van den Oever, Med. Sci. Law., 16 (1976) 269.

[5] B. Knight, Simpson's Forensic Medicine, Hodder Arnold Publications, New York, 1997.

[6] L. Swann, G.E. Chidlow, S. Forbes, S.W. Lewis, J. Forensic Sci., 55 (2010) 308.

[7] L. Swann, S. Forbes, S.W. Lewis, Aust. J Forensic Sci. (2009).

[8] R.I. Mackie, P.G. Stroot, V.H. Varel, J. Anim. Sci., 76 (1998) 1331.

[9] B.B. Dent, S.L. Forbes, B.H. Stuart, Environ. Geol., 45 (2004) 576.

[10] N. Anastos, N.W. Barnett, S.W. Lewis, Talanta, 67 (2005) 269.

[11] S. Oguri, J. Chromatogr. B., 747 (2000) 1.

[12] H.J. Isaaq, Electrophoresis, 20 (1999) 3190.

[13] R.L. Cunico, K.M. Gooding, T. Wehr, Basic HPLC and CE of Biomolecules, Bay Bioanalytical Laboratory, Inc., Richmond, California, 1998.

[14] G. Nouadje, N. Simeon, F. Dedieu, M. Nertz, P. Puig, F. Couderc, J. Chromatogr. A, 765 (1997) 337. 
[15] M. Krizek, T. Pelikanova, J. Chromatogr. A, 815 (1998) 243.

[16] F. Kvasnicka, M. Voldrich, J. Chromatogr. A, 1103 (2006) 145.

[17] N. Zhang, H. Wang, Z.-X. Zhang, Y.-H. Deng, H.-S. Zhang, Talanta, 76 (2008) 791.

[18] Y.M. Liu, J.K. Cheng, J. Chromatogr. A, 1003 (2003) 211.

[19] Q. Wang, H. Yu, H. Li, F. Ding, P. He, Y. Fang, Food Chem, 83 (2003) 311.

[20] S. Cortacero-Ramirez, A. Segura-Carretero, C. Cruces-Blanco, M. RomeroRomero, A. Fernandez-Gutierrez, Anal. Bioanal. Chem., 380 (2004) 831.

[21] W.C. Lin, C.E. Lin, E.C. Lin, J. Chromatogr. A, 755 (1996) 142.

[22] K.G. Schoenly, N.H. Haskell, D.K. Mills, C. Bieme-Ndi, K. Larsen, Y. Lee, American Biology Teacher, 68 (2006) 402.

[23] D.L. France, Griffin, T.J., Swanburg, J.G., Lindermann, J.W., Davenport, G.C., Trammell, V. , J. Forensic Sci., 37 (1992) 1445.

[24] S. Orlandini, L. Giannini, S. Pinzauti, S. Furlanetto, Talanta, 74 (2008) 570.

[25] L.R. Snyder, J.L. Glajch, J.J. Kirkland, Practical HPLC Method Development, John Wiley and Sons, New York, 1988.

[26] V.M. Morris, J.G. Hughes, P.J. Marriott, J. Chromatogr. A, 755 (1996) 235.

[27] D.N. Heiger, Capillary Electrophoresis - An Introduction, Hewlett-Packard Company, France, 1992.

[28] R.G. Brereton, Applied Chemometrics for Scientists, Wiley and Sons Ltd, England, 2007.

[29] J. Lange, K. Thomas, C. Wittmann, J. Chromatogr. B, 779 (2002) 10. 
Table 1. Selected biogenic amines. * indicates successful separation by method outlined in this work

\begin{tabular}{|c|c|c|}
\hline Name & Structure & pKa [Ref] \\
\hline Histamine & & $9.75[24]$ \\
\hline Tryptamine* & & $10.2[24]$ \\
\hline Tyramine* & & $9.74[24]$ \\
\hline Putrescine & & $9.35[24]$ \\
\hline Cadaverine & & 10.05 [24] \\
\hline Phenylalanine* & & $9.31[24]$ \\
\hline Benzylamine* & & $9.33[25]$ \\
\hline Aniline* & & $4.63[25]$ \\
\hline Tryptophan* & & $9.41[24]$ \\
\hline Tyrosine* & & $9.21[24]$ \\
\hline Indole & & $16.7[25]$ \\
\hline
\end{tabular}


Table 2: Levels of the four design variables as used in the screening design. (-1) indicates the low level, $(+1)$ indicates the high level. Table 1 also details the components of the running buffers.

\begin{tabular}{|l|l|l|l|l|l|}
\hline Experiment & $\mathbf{p H}$ & {$[$ BGE] [20] } & $\begin{array}{l}\text { \% Methanol } \\
(\mathbf{v} / \mathbf{v})\end{array}$ & Voltage & CEF \\
\hline 1 & $9(-1)$ & $50(-1)$ & $20(-1)$ & $20(-1)$ & 591.3 \\
\hline 2 & 9 & 50 & 20 & $30(+1)$ & 512.9 \\
\hline 3 & 9 & 50 & $40(+1)$ & 20 & 13.9 \\
\hline 4 & 9 & 50 & 40 & 30 & 11.5 \\
\hline 5 & 9 & $100(+1)$ & 20 & 20 & 1141.3 \\
\hline 6 & 9 & 100 & 20 & 30 & 983.1 \\
\hline 7 & 9 & 100 & 40 & 20 & 15.6 \\
\hline 8 & 9 & 100 & 40 & 30 & 12.5 \\
\hline 9 & $10(+1)$ & 50 & 20 & 20 & 2544.2 \\
\hline 10 & 10 & 50 & 20 & 30 & 687.9 \\
\hline 11 & 10 & 50 & 40 & 20 & 8721.3 \\
\hline 12 & 10 & 50 & 40 & 30 & 2716.1 \\
\hline 13 & 10 & 100 & 20 & 20 & 16152.8 \\
\hline 14 & 10 & 100 & 20 & 30 & 11238 \\
\hline 15 & 10 & 100 & 40 & 20 & 12490.4 \\
\hline 16 & 10 & 100 & 40 & 30 & 15916.4 \\
\hline
\end{tabular}


Table 3: p-values for individual factors and all interactions showing statistical significance.

\begin{tabular}{|c|c|}
\hline Individual Factors and Interactions & p-value (* indicates a significant effect) \\
\hline $\mathrm{pH}$ & $0.0013 *$ \\
\hline [BGE] & $0.0106^{*}$ \\
\hline$\%$ Organic Modifier & 0.6378 \\
\hline Voltage & 0.4382 \\
\hline $\mathrm{pH} *[\mathrm{BGE}]$ & $0.0119^{*}$ \\
\hline $\mathrm{pH}^{* \%} \%$ Organic Modifier & $0.0285^{*}$ \\
\hline pH*Voltage & 0.4191 \\
\hline$[\mathrm{BGE}] * \%$ Organic Modifier & 0.5102 \\
\hline$[\mathrm{BGE}]^{*}$ Voltage & 0.6223 \\
\hline \% Organic Modifier*Voltage & 0.7443 \\
\hline $\mathrm{pH}^{*}[\mathrm{BGE}] * \%$ Organic Modifier & 0.5761 \\
\hline pH*[BGE]*Voltage & 0.5596 \\
\hline $\mathrm{pH}^{* \%} \%$ Organic Modifier*Voltage & 0.7161 \\
\hline$[\mathrm{BGE}] * \%$ Organic Modifier*Voltage & 0.2535 \\
\hline pH*[BGE]*\% Org Modifier*Voltage & 0.2875 \\
\hline
\end{tabular}


Table 4: Coded three-factor central composite design consisting of three levels, detailing star points and replication.

\begin{tabular}{|c|c|c|c|}
\hline & $\mathbf{p H}$ & [BGE] & $\begin{array}{c}\text { \% Organic } \\
\text { Modifier }\end{array}$ \\
\hline \multirow[t]{8}{*}{ Full factorial } & -1 & -1 & -1 \\
\hline & -1 & -1 & +1 \\
\hline & -1 & +1 & -1 \\
\hline & -1 & +1 & +1 \\
\hline & +1 & -1 & -1 \\
\hline & +1 & -1 & +1 \\
\hline & +1 & +1 & -1 \\
\hline & +1 & +1 & +1 \\
\hline \multirow[t]{7}{*}{ Star Points } & 0 & 0 & 0 \\
\hline & 0 & 0 & -1.68 \\
\hline & 0 & 0 & +1.68 \\
\hline & 0 & -1.68 & 0 \\
\hline & 0 & +1.68 & 0 \\
\hline & -1.68 & 0 & 0 \\
\hline & +1.68 & 0 & 0 \\
\hline \multirow[t]{5}{*}{ Replication } & 0 & 0 & 0 \\
\hline & 0 & 0 & 0 \\
\hline & 0 & 0 & 0 \\
\hline & 0 & 0 & 0 \\
\hline & 0 & 0 & 0 \\
\hline
\end{tabular}


Table 5: Peak identification and migration times (mins) for standard mixture containing eight biogenic amines and amino acids at pre- and post-optimisation conditions by capillary electrophoresis.

\begin{tabular}{|l|l|l|l|l|}
\hline Peak & Peak Identification & $\begin{array}{l}\text { Migration } \\
\text { Time (mins) } \\
\text { Pre- } \\
\text { Optimisation }\end{array}$ & $\begin{array}{l}\text { Migration } \\
\text { Time (mins) } \\
\text { Post- } \\
\text { Optimisation }\end{array}$ & $\begin{array}{l}\text { Migration } \\
\text { time RSD } \\
(\mathbf{\%}) \\
(\mathbf{n}=\mathbf{1 0})\end{array}$ \\
\hline 1 & Tryptamine & 3.895 & 4.760 & 2.0 \\
\hline 2 & Tyramine & 4.197 & 5.279 & 2.6 \\
\hline 3 & Benzylamine/Aniline & 4.341 & 5.498 & - \\
\hline 4 & Neutral Marker & 5.584 & 6.607 & - \\
\hline 5 & Tryptophan & 6.974 & 9.861 & 3.9 \\
\hline 6 & Tyrosine & 7.525 & 10.971 & 4.3 \\
\hline 7 & Phenylalanine & 7.865 & 11.773 & 4.3 \\
\hline
\end{tabular}


Table 6: Calibration data for selected biogenic amines

\begin{tabular}{|l|l|l|l|}
\hline Analyte & Calibration equation & $\mathbf{r}^{2}$ & $\mathbf{L O D}^{\mathbf{a}} \mathbf{( m g / L )}$ \\
\hline Tryptamine & $y=0.1100 x+0.1701$ & 0.9965 & 5.9 \\
\hline Tyramine & $y=0.1393 x+0.3306$ & 0.9967 & 5.8 \\
\hline Tryptophan & $y=0.278 x+0.8537$ & 0.9959 & 6.5 \\
\hline Tyrosine & $y=0.255 x+0.764$ & 0.9935 & 8.2 \\
\hline Phenylalanine & $y=0.227 x+0.7494$ & 0.9929 & 8.6 \\
\hline
\end{tabular}

${ }^{\text {a }}$ Limit of detection: calculated as the analyte concentration that gave a signal equal to the blank signal plus three standard deviations of the blank [32]. 